\title{
Comparative and Behavioral Study on VANET Routing Protocols
}

\author{
S.Sujatha ${ }^{1}$, P.Soundeswari ${ }^{2}$ \\ ${ }^{1}$ (Associate Professor, Computer Science, Dr.G.R.D College of Science, India) \\ ${ }^{2}$ (Scholar, Computer Science, Dr.G.R.D College of Science, India)
}

\begin{abstract}
Vehicular ad hoc networks (VANETs) without any support from fixed infrastructure offer a large number of applications. A Complete understanding of the communications Channel between vehicles is necessary for realistic modeling of VANETs and the development of related technologies and applications. This paper gives a brief summary of different routing algorithms in VANET and also discusses the major classifications. The comparative study is mainly done on the essential characteristics and behavior of the protocols and based on the comparison results, the position based protocol is considered as the best for handling issues in packet delay, traffic congestion and better throughput in Vehicular ad hoc networks.
\end{abstract}

Keywords: VANET, CAR, CGSR, DSR, DV-CAST

\section{Introduction}

VANET stands for vehicular ad hoc network provide better communication between moving vehicles and fixed equipments, VANET is a new standard that integrates Wi-Fi, Bluetooth and other mobile connectivity protocols. The essential requirement of VANET is that it should be able to communicate in any environment irrespective of traffic densities and vehicle locations. Vehicular communications are made in fluctuating environment and should work both in urban and rural areas.

The primary factors to be considered in designing a VANET is security, location and the maintenance of the services. As an open network VANET is always targeted to malicious communication, the safety and security factors of the messages should not harm the traffic and driver. MANET routing protocol is not suitable for VANET because MANET routing protocol has difficulties from finding stable routing paths in VANET environments. Six types of routing protocols in VANETs are topology based, position based, geocast based, cluster based, broadcast based and infrastructure based.

The rest of the paper is organized as follows. Section 2 deals topology based protocols, section 3 on position based protocols, section 4 on broadcast protocols, section 5 on performance study and comparison results on VANET Protocols, section 6 concludes the paper.

\section{Topology Based Protocols}

These protocols discover the route and maintain it in a table before the sender starts transmitting data. They are further divided into reactive, proactive and hybrid protocols.

\subsection{Proactive protocols}

The proactive protocol is also known as table driven routing protocol. These protocols work by periodically exchanging the knowledge of topology among all the nodes of the work. The proactive protocols do not have initial route discovery delay but consumes lot of bandwidth for periodic updates of topology. There are several routing protocols that fall under this category

\subsubsection{Source-Tree Adaptive Routing (STAR)}

Source-Tree Adaptive Routing (STAR) is another link State protocol. It reduces overhead on the network by eliminating periodic updates. This protocol can be suitable for large scale networks but it needs large memory and processing because it has to maintain large trees for whole network. The Each node maintains a source tree. Each node builds a partial topology graph using aggregates of neighbor information learnt using an underlying neighbor discovery protocol and source trees reported by the neighbors [1].

2.1.2 Clusterhead Gateway Switch Routing (CGSR)

CGSR is a clustered multihop mobile wireless network with several heuristic routing schemes. Advantages of CGSR Better bandwidth utilization reduce the size of distance vector table because the routing is performed only over cluster head. Disadvantages of CGSR More time is spend in selection of cluster heads and gateways if the mobile node uses CDMA/TDMA then it can take some time to get permission to send packets Changes in the cluster-head, may result in multiple path breaks [2].

2.1.3 Destination Sequenced Distance Vector Routing (DSDV) 
Each DSDV node maintains two routing tables: one for forwarding packets and one for advertising incremental routing packets. Advantages of DSDV It is quite suitable for creating ad-hoc networks with small number of nodes Solve the Routing Loop problem Count to infinity problem is reduced DSDV maintains only the best path instead of maintaining multiple paths to every destination . Disadvantages of DSDV, DSDV requires a regular update of its routing tables, which uses up battery power and a small amount of bandwidth even when the network is idle whenever the topology of the network changes, a new sequence number is necessary DSDV is not suitable for highly dynamic networks [3].

2.2 Reactive protocols

Reactive routing opens a route only when it is necessary for a node to communicate with another node. It maintains only the routes that are currently in use, thereby reducing the burden on the network.

\subsubsection{Dynamic source routing (DSR)}

This protocol consists of two operations "Route Discovery" and "Route Maintenance" that makes it self-configuring and self-organizing. Another important property of DSR routing protocol is network type flexibility. Disadvantages of DSR The route maintenance mechanism does not locally repair a broken link The connection setup delay is higher than in table-driven protocols This routing overhead is directly proportional to the path length [3].

\subsubsection{Dynamic MANET On demand (DYMO)}

DYMO is another reactive routing protocol that works in multi hop wireless networks. DYMO has a simple design and is easy to implement. The basic operations of DYMO protocol are route discovery and route Maintenance was studied extensively along with comparison of two on demand routing protocols [4].

\section{Position Based Routing Protocols}

Position based routing consists of class of routing algorithm. Position based routing is beneficial since no global route from source node to destination node need to be created and maintained. Position Based routing is broadly divided in two types: Position based greedy V2V protocols, Delay Tolerant Protocols.

3.1 Greedy forwarding protocol

3.1.1 Geographic Source Routing (GSR)

GSR use "switch back to greedy" method for local recovery. After a packet reach to its local maximum, it switch back to greedy forwarding. The sender node reaches the destination by using the road topology map and the above information. In other words in GSR the source node finds the shortest path to destination on the graph using simple graph algorithms and marks the packet with destination's location. In this the packet travels through junctions to reach the destination. In this algorithm, each node maintains a Neighbor list, a Topology table, a Next Hop table and a Distance table [1].

3.1.2 Anchor-Based Street and Traffic Aware Routing (A-STAR)

A-STAR is traffic aware: the traffic on the road determines whether the anchor points of the road will be considered in the shortest path. A-STAR routes based on two kinds of overlaid maps: a statically rated map and a dynamically rated map. A statistically rated map is a graph that displays bus routes that typically imply stable amount of traffic. The development of A-STAR was inconsideration with city environment. A-STAR also use traffic information and street awareness in path finding [5].

\subsubsection{Greedy Perimeter coordinator Routing (GPCR)}

GPCR (Greedy Perimeter coordinator Routing) modified to adapt to city scenario. Here, a restrictive greedy algorithm is simply followed when nodes are in street and an actual routing decision is taken when at the junction of streets. Here the packet is forwarded to a node in the junction rather sending it across the junction. GPCR traverses the junctions by a restricted greedy forwarding procedure [6].

3.1.4 Vehicle-Assisted Data Delivery Routing Protocol (VADD)

VADD protocol adopted the idea of carry-and-forward for data delivery from a moving vehicle to a static destination. Use predictable traffic pattern and vehicle mobility to assist efficient data delivery. A vehicle makes a decision at a junction and selects the next forwarding path with the smallest packet delivery delay. A path is simply a branched road from an intersection. It is suitable for multi-hop data delivery [5].

\subsubsection{Connectivity Aware Routing Protocols (CAR)}

CAR is designed specifically for inter-vehicle communication in a city and/or highway environment. CAR integrates locating destinations with finding connected paths between source and destination.CAR ensures to find the shortest connected path because CAR has higher packet delivery ratio than GPSR and GPSR+AGF. It cannot adjust with different sub-path when traffic environment changes [7].

\subsubsection{Diagonal-Intersection-Based Routing Protocol (DIR)}


DIR protocol constructs a series of diagonal intersections between the source and destination vehicle. DIR vehicle is auto adjustable, Auto adjustability means that one sub path with low data packet delay between two neighboring diagonal intersections, which is dynamically selected to forward data packets. For given a pair of neighboring diagonal intersections, two or more disjoint sub-paths exist between them. To reduce the data packet delay, the route is automatically re-routed by the selected sub-path with lowest delay [8].

\subsubsection{Border-node based most forward within radius routing protocol (B-MFR)}

Border-node based Most Forward within Radius routing (B-MFR) which uses the concept of border-node within the sender's communication range to minimize the number of hops between source and destination. The B-MFR utilizes the border-node to avoid using interior nodes within the transmission range for further transmitting the packet. Next-hop forwarding method like greedy forwarding scheme for linear network does not support well in highly mobile ad hoc network such as vehicular ad hoc network [9].

\subsubsection{GVGrid}

GVGrid is designed not for sparse regions with high-speed vehicles such as highways, but for dense regions with low-speed vehicles such as cities. It also reconstructs the route when it is broken by the movement of vehicles. GVGrid divides the geographical area into uniform-size squares called grids. Then the intermediate grids between source and destination are recorded in the routing table. An appropriate vehicle which has the fewest number of disconnections in each grid is chosen to forward packets to next grid. GVGrid consists of two processes, a route discovery process and a route maintenance process [10].

\subsubsection{Contention Based Forwarding (CBF)}

The contention-based forwarding (CBF) algorithm is a greedy position-based forwarding algorithm that does not require the proactive transmission of beacon messages. In CBF, the next hop is selected through a distributed contention process based on the actual positions of all of the current neighbors. In this contention process, CBF makes use of biased timers. To avoid packet duplication, the first node that is selected suppresses the selection of further nodes using an area-based suppression algorithm [11].

\subsubsection{Directional Greedy Routing Protocol (DGRP)}

DGRP is a position based greedy routing protocol, which uses the location, speed and direction of motion of their neighbors to select the most appropriate next forwarding node. . It predicts the position of nodes within the beacon interval whenever it needs to forward a data packet. This prediction can be done using previous known position, speed, and direction of motion of node [12].

\subsubsection{Predictive Directional Greedy Routing Protocol (PDGRP)}

Jiayu Gong proposed PDGRP, in which the weighted score is calculated from two strategies namely, position first forwarding and direction first forwarding. Here next hop selection is done on prediction and it is not reliable at all situations. It doesn't guarantee the delivery of packet to the node present in the edge of the Transmission range of forwarding node, which is considered as most suitable next hop, due to high dynamics of vehicles. This will lead to low packet delivery ratio, high end to end delay and increased routing overhead [13].

\subsubsection{Hierarchical Clustering Based Greedy Routing (HCBGR)}

HCBGR is a unicast position based greedy routing algorithm designed for sending messages from any node to any other node in VANETs. The HCBGR Algorithm has six basic functional units. The first is Neighbor Node Identification(NNI), the second is Distance Calculation(DC), the third is Direction of Motion Identification(DMI), the fourth is Reckoning Link Stability(RLS), the fifth is Weighted score calculation(WS) and the sixth is Potential Node Selection(PNS) [13].

3.1.13 Reliable Directional Greedy Routing (RDGR)

Reliable Directional Greedy Routing (RDGR) is a reliable position-based greedy routing approach which uses the position, speed, direction of motion, and link stability of neighbors to select the most appropriate next forwarding node. The packet sender or forwarder node, selects neighbor nodes which have forward progress towards destination node using velocity vector, and checks link stability of those nodes [14].

\section{Broadcasting Based Routing Protocols}

Broadcast routing is frequently used in VANET for sharing, traffic, weather and emergency, road Conditions among vehicles and delivering advertisements and announcements. Broadcasting is used when message needs to be disseminated to the vehicle beyond the transmission range i.e. multi hops are used. 
Comparative And Behavioral Study On VANET Routing Protocols

4.1 Distributed vehicular broadcast protocol (DV-CAST)

DV-CAST for a multi-hop broadcast routing protocol in VANETs and indicates three traffic scenarios for a vehicular broadcasting; dense traffic scenario, sparse traffic scenario, and regular traffic scenario. This protocol causes high control overhead and delay in end to end data transfer [10].

\subsection{Preferred group broadcast (PGB)}

PGB is not a reliable broadcasting protocol but it is a solution to prevent broadcast storm problem from route request broadcasting. Each node in PGB will sense the level of signal strength from neighbor broadcasting. The signal strength is used for waiting timeout calculation. Nodes in the edge of circulated broadcast will set shorter waiting timeout. Only node with shortest timeout will rebroadcast the message. But there exists a problem on low density area [15].

\section{Performance Study on VANET Protocols}

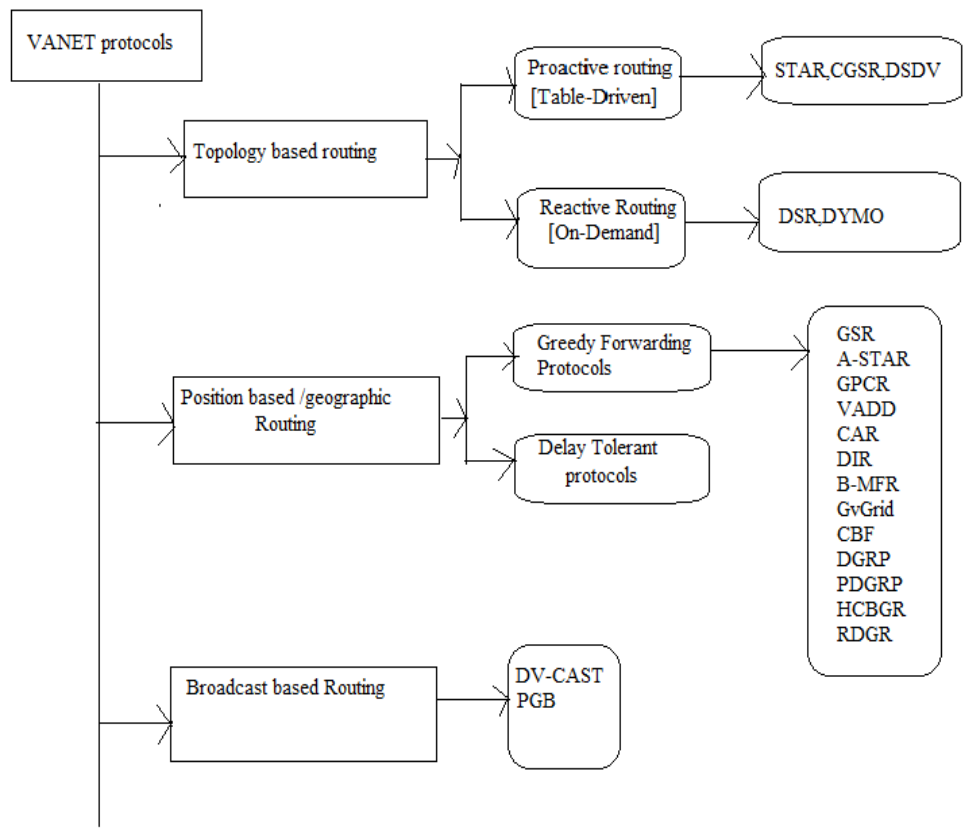

Fig. 1 VANET protocols

TABLE 1 Comparison Results of routing protocols in VANETs

\begin{tabular}{|l|l|l|l|l|l|}
\hline Protocol & Scenario & Routing maintenance & Routing type & Strategy & Simulator \\
\hline STAR & Urban & Proactive & Unicast & Greedy forwarding & NS2 \\
\hline CGSR & Urban & Proactive & Unicast & Multihop & NS2 \\
\hline DSDV & Urban & Proactive & Unicast & Multihop & NS3 \\
\hline DSR & Urban & Reactive & Unicast & Multihop & NS2 \\
\hline DYMO & Urban & Reactive & Unicast & Multihop & QUALNET \\
\hline GSR & Urban & Reactive & Unicast & Greedy forwarding & NS2 \\
\hline A-STAR & Urban & Reactive & Unicast & Greedy forwarding & NS2 \\
\hline GPCR & Urban & Reactive & Unicast & Greedy forwarding & NS2 \\
\hline VADD & Urban & Reactive & Unicast & multihop & NS2 \\
\hline CAR & Urban & Reactive & Unicast & Greedy forwarding & NS2 \\
\hline DIR & Urban & Reactive & Unicast & Greedy forwarding & NS2 \\
\hline B-MFR & Urban & Reactive & Unicast & Greedy forwarding & NS2 \\
\hline GVGrid & Urban & Reactive & Unicast & Greedy forwarding & NS2 \\
\hline CBF & Urban & Reactive & Unicast & Greedy forwarding & NS2 \\
\hline DGRP & Urban & Reactive & Unicast & Greedy forwarding & NS2 \\
\hline PDGRP & Urban & Reactive & Unicast & Greedy forwarding & NS2 \\
\hline HCBGR & Urban & Reactive & Unicast & Greedy forwarding & NS2 \\
\hline RDGR & Urban & Reactive & Unicast & Greedy forwarding & NS2 \\
\hline DV-CAST & Highway & Proactive & Broadcast & Multihop & NS2 \\
\hline PGB & Urban & Reactive & Unicast & Multihop & NS2 \\
\hline
\end{tabular}


This table gives a comparison of these protocols and discusses forward strategy, routing type, protocol scenario, routing maintenance and network simulator of the different VANET routing protocols. Among this scenario the position based routing protocol works best in open space scenario with evenly distributed nodes. The absence of fewer obstacles in highway scenario is attributed to its good performance. From forward strategies position based routing technique employs the awareness of vehicle about the position of other vehicle to develop routing strategy.

\section{Conclusion}

In this paper we have discussed several VANET protocols. Position of the vehicle is one of the most important data for vehicles. Position based routing protocols need the information about the physical location of the participating vehicles to be made available. After analyzing the survey of protocols, it is found that the position based routing has better performance because there is no creation and maintenance of global route from source node to destination node. In the position based routing protocol, all the packets are received with small average delay, better throughput, and effective utilization and also helps to prevent the accidents on the road effectively. In future these protocols can also be used for further research in VANET.

\section{References}

[1] Bilal Mustafa, Umar Waqas Raja," Issues of Routing in VANET”, Master Thesis, Computer Science, C m p t S i, and Thesis no: MCS-2010-20 Jun 2010.

[2] Natarajan Meghanathan", "Survey and taxonomy of unicast routing protocols for mobile ad hoc networks", The International Journal on Applications of Graph Theory in Wireless Ad hoc Networks and Sensor Networks (GRAPH-HOC), Vol.1, No.1, December 2009.

[3] Pankaj barodia, "routing protocols in wireless network", Deenbandhu Chhotu Ram University of Science and Technology Murthal, Sonipat (Haryana), June 2011.

[4] Manish Sharma ${ }^{1} \&$ Gurpadam Singh,"Evaluation of Proactive, Reactive and Hybrid Ad hoc Routing Protocol for various Battery models in VANET using Qualnet"

[5] Uma Nagaraj, ' ${ }^{2}$ Dr. M. U. Kharat, ${ }^{3}$ Poonam Dhamal,"Study of Various Routing Protocols in VANET", IJCST Vol. 4, ISSue 4, oCT.-DeC. 2011.

[6] Arnab Kumar Banik,"Routing Protocol with prediction based mobility model in vehicular ad hoc network (VANET)", April 2010.

[7] Yuh-Shyan Chen,"Routing in Vehicular Ad Hoc Networks (VANETs)",NTPU, Department of Computer Science and Information Engineering.

[8] Rakesh Kumar, ${ }^{2}$ Mayank Dave," A Comparative Study of Various Routing Protocols in VANET",IJCSI International Journal of Computer Science Issues, Vol. 8, Issue 4, No 1, July 2011.

[9] Monika, Sanjay Batish and Amardeep Singh," Border-node based Movement Aware Routing Protocol", International Journal of Computer Science and Informatics ISSN (PRINT): 2231 -5292, Vol-1, Iss-4, 2012.

[10] YUN-WEI LIN', YUH-SHYAN CHEN ${ }^{2}$, AND SING-LING LEE," Routing Protocols in Vehicular Ad Hoc Networks: Survey and Future Perspectives".

[11] Si-Ho Cha ${ }^{1}$, Min-Woo Ryu ${ }^{2}$ and Kuk-Hyun Cho,"A Survey of Greedy Routing Protocols for Vehicular Ad Hoc Networks", Smart Computing Review, Vol. 2, no. 2, April 2012|25.

[12] K. Jayasudha," Hierarchical Clustering Based Greedy Routing in Vehicular Ad Hoc Networks", European Journal of Scientific Research ISSN 1450-216X Vol.67 No.4 (2012), pp. 580-594 @ Euro Journals Publishing, Inc. 2012

[13] K. Lakshmi ${ }^{1}$, K.Thilagam ${ }^{2}$, K. Rama ${ }^{3}$, A.Jeevarathinam ${ }^{4}$, S. Manju Priya ${ }^{5}$," Comparison of Three Greedy Routing Algorithms for Efficient Packet Forwarding in VANET", IJCT A|JAN-FEB 2012.

[14] K.Prasanth ${ }^{1}$ Dr. K.Duraiswamy ${ }^{2}$ K.Jayasudha ${ }^{3}$ and Dr.C.Chandrasekar,"I m p r o v e d P a c k e t F o r w a r d i n g A p p r o a c h i n V A N E T u s i n g R D G R a 1 g o r i t h m ", International Journal of Next Generation Network (IJNGN), Vol.2, No.1, March 2010.

[15] Uma Nagaraj, Poonam Dhamal, "Broadcasting Routing Protocols in VANET", ISSN 2224-610X (Paper) ISSN 2225-0603 (Online) Vol 1, No.2, 2011. 